PREPARED FOR SUBMISSION TO JHEP

\title{
On Partially Massless Bimetric Gravity
}

\author{
S.F. Hassan, Angnis Schmidt-May Mikael von Strauss \\ Department of Physics $\&$ The Oskar Klein Centre, \\ Stockholm University, AlbaNova University Centre, SE-106 91 Stockholm, Sweden \\ E-mail: fawad@fysik.su.se, angnis.schmidt-may@fysik.su.se, \\ mvs@fysik.su.se
}

AbStRact: We extend the notion of the Higuchi bound and partial masslessness to ghostfree nonlinear bimetric theories. This can be acheived in a simple way by first considering linear massive spin-2 perturbations around maximally symmetric background solutions, for which the linear gauge symmetry at the Higuchi bound is easily identified. Then, requiring consistency between an appropriate subset of these transformations and the dynamical nature of the backgrounds, fixes all but one parameter in the bimetric interaction potential. This specifies the theory upto the value of the Fierz-Pauli mass and leads to the unique candidate for nonlinear partially massless bimetric theory.

KEYWORDS: modified gravity, massive gravity, higher spin fields

\section{Contents}

1 Introduction $\quad 2$

2 The Higuchi bound and partial masslessness in linear massive gravity 3

3 De Sitter solutions and their perturbations in bimetric theory 3

4 Determination of the partially massless bimetric theory 5

$\begin{array}{lll}5 & \text { Nonlinear scaling symmetry } & 7\end{array}$

6 Summary and Discussions $\quad 8$ 


\section{Introduction}

Fierz and Pauli (FP) obtained a ghost-free linear theory of massive spin-2 fields in flat space [1, $2]$. When considered in de Sitter backgrounds, the theory has interesting features associated with the Higuchi bound, $m_{\mathrm{FP}}^{2}=\frac{2}{3} \Lambda$, that determines the mass of the spin- 2 state in terms of the cosmological constant $[3,4]$. A new gauge symmetry appears at the bound and eliminates the longitudinal mode of the spin-2 field, leaving behind only 4 propagating modes [5-11]. The resulting theory is the linear partially massless theory on a de Sitter background.

To better understand the origin and consequences of this symmetry, one needs to work with a fully dynamical, nonlinear version of the FP theory. On general grounds, such nonlinear massive spin-2 theories require working with two metrics, say, $g_{\mu \nu}$ and $f_{\mu \nu}$. But they are also generically plagued by the Boulware-Deser ghost instability $[12,13]$. A major breakthrough in this field was the work of $[14,15]$. Here, the authors developed the nonlinear massive gravity for a flat reference metric $f_{\mu \nu}=\eta_{\mu \nu}$, the dRGT model, and established that it was ghost free in a certain "decoupling limit" analysis $[16,17]$ that proved to be very powerful for the purpose. All subsequent developments in the field are based on this breakthrough. However, the decoupling limit analysis cannot show the absence of ghost away from this specific limit. That the model remained ghost-free at the complete non-linear level was proven in [18].

As such the dRGT model does not admit de Sitter solutions since it was constructed for $f_{\mu \nu}=\eta_{\mu \nu}$, to which the decoupling limit analysis is mostly confined. However, the nonlinear analysis of [18] could be used to prove that massive gravity with generic non-dynamical reference metric $f_{\mu \nu}$ [19] was ghost-free at the completely non-linear level [20]. Finally the bimetric theory was obtained and proven to be ghost-free in [21, 22]. This theory admits completely dynamical de Sitter solutions with massive spin-2 fluctuations around them. Hence it provides a natural setup for investigating partial masslessness and the associated symmetries.

It is now interesting to ask whether there exists a nonlinear extension of the linear partially massless FP gravity within the family of ghost-free massive gravity and bimetric theories. In the context of massive gravity, this question was recently investigated in [23], in a decoupling limit specifically developed for the de Sitter space. The authors discover the parameter values for which the Stückelberg field that captures the dynamics of the helicity-zero mode of the graviton is removed to all orders in the decoupling limit. If this result extends beyond the decoupling limit, then the theory has a nonlinear gauge symmetry that removes the helicity-zero excitation, and thus constitutes the nonlinear partially massless theory of gravity . For a related investigation, see [24].

Here we investigate the problem in the ghost-free bimetric setup where the backgrounds

are not fixed by hand but arise dynamically. A large class of such theories can satisfy the Higuchi bound and exhibit linear partial masslessness. It is shown that by demanding consistency between the dynamical backgrounds and just a subset of the linear gauge transformations known from the FP theory, one can arrive at a unique non-linear candidate for a partially massless bimetric theory.

Other recent works on partial masslessness, but in somewhat different setups, include 
$[25,26]$, as well as [27] in the "new massive gravity" framework of [28, 29]. The issue arises also in the context of higher-spin theories (see e.g. [30-32]).

\section{The Higuchi bound and partial masslessness in linear massive gravity}

The linear Fierz-Pauli equation for a massive graviton on a fixed de Sitter background reads,

$$
\overline{\mathcal{E}}_{\mu \nu}^{\rho \sigma} h_{\rho \sigma}-\Lambda\left(h_{\mu \nu}-\frac{1}{2} \bar{g}_{\mu \nu} \bar{g}^{\rho \sigma} h_{\rho \sigma}\right)+\frac{m_{\mathrm{FP}}^{2}}{2}\left(h_{\mu \nu}-\bar{g}_{\mu \nu} \bar{g}^{\rho \sigma} h_{\rho \sigma}\right)=0 .
$$

$\Lambda$ is the cosmological constant and $m_{F P}$ is the mass of the spin-2 fluctuation. $\bar{g}_{\mu \nu}$ is the de Sitter metric and the kinetic operator is given by,

$$
\overline{\mathcal{E}}_{\mu \nu}^{\rho \sigma} h_{\rho \sigma}=-\frac{1}{2}\left[\delta_{\mu}^{\rho} \delta_{\nu}^{\sigma} \bar{\nabla}^{2}+\bar{g}^{\rho \sigma} \bar{\nabla}_{\mu} \bar{\nabla}_{\nu}-\delta_{\mu}^{\rho} \bar{\nabla}^{\sigma} \bar{\nabla}_{\nu}-\delta_{\nu}^{\rho} \bar{\nabla}^{\sigma} \bar{\nabla}_{\mu}-\bar{g}_{\mu \nu} \bar{g}^{\rho \sigma} \bar{\nabla}^{2}+\bar{g}_{\mu \nu} \bar{\nabla}^{\rho} \bar{\nabla}^{\sigma}\right] h_{\rho \sigma} .
$$

The mass term breaks the symmetry under infinitesimal reparameterizations.

It is known that, in this theory, a curious role is played by the Higuchi bound,

$$
m_{\mathrm{FP}}^{2}=\frac{2}{3} \Lambda \text {. }
$$

Above the bound, $m_{\mathrm{FP}}^{2}>\frac{2}{3} \Lambda_{g},(2.1)$ propagates only the five healthy polarizations of the massive spin-2 fluctuation. In fact, the mass term is fixed uniquely by demanding that a sixth ghost mode decouples $[1,2]$. Below the bound, $m_{\mathrm{FP}}^{2}<\frac{2}{3} \Lambda_{g}$, the helicity zero component of the spin-2 field becomes a ghost and the theory becomes unstable [3, 4]. But precisely at the Higuchi bound, (2.1) develops a new gauge symmetry that decouples the helicity zero component, leaving only four healthy propagating modes [5-10]. The theory with this value for the mass is often referred to as partially massless.

The new linear gauge symmetry that emerges at the Higuchi bound reads [5],

$$
h_{\mu \nu} \longrightarrow h_{\mu \nu}+\delta h_{\mu \nu} \quad \text { with } \quad \delta h_{\mu \nu} \equiv\left(\nabla_{\mu} \nabla_{\nu}+\frac{\Lambda}{3} \bar{g}_{\mu \nu}\right) \xi(x)
$$

where, $\xi(x)$ is an arbitrary gauge transformation parameter. Note that the solutions of $\delta h_{\mu \nu}=0$ give conformal Killing transformations $\delta x^{\mu}=\nabla^{\mu} \xi$ on de Sitter space. These are excluded since the FP theory has no coordinate invariance.

Understanding the origin of (2.4) and partial masslessness requires a nonlinear version of the FP theory that, furthermore, treats the background dynamically. This suggests working with the ghost-free bimetric theory that will be reviewed in the next section and which will be shown to provide a natural setup for addressing the issue of partial masslessness.

\section{De Sitter solutions and their perturbations in bimetric theory}

The most general bimetric action with the correct combination of the kinetic and potential terms that avoids the Boulware-Deser ghost at the nonlinear level is given by, [21, 22]

$$
S_{g f}=\int \mathrm{d}^{4} x\left[m_{g}^{2} \sqrt{g} R(g)+m_{f}^{2} \sqrt{f} R(f)-2 m^{4} \sqrt{g} \sum_{n=0}^{4} \beta_{n} e_{n}\left(\sqrt{g^{-1} f}\right)\right] .
$$


The $e_{n}(S)$ are the elementary symmetric polynomials of the eigenvalues of the matrix $S$. The action has seven independent parameters: the Planck masses $m_{g}$ and $m_{f}$, and the five dimensionless $\beta_{n}$. The mass scale $m$ is degenerate with these. The potential is an extension of the 3-parameter massive gravity potential [15] in the formulation of [19].

The equations of motion for $g_{\mu \nu}$ and $f_{\mu \nu}$, obtained from (3.1) are given, for example, in [33] on which the present section is based. To relate to the Higuchi bound, we are interested in de Sitter solutions. Generic de Sitter solutions for both of the bimetric equations are of the type, ${ }^{1}$

$$
\bar{f}_{\mu \nu}=c^{2} \bar{g}_{\mu \nu},
$$

where $c$ is a constant, generically determined in terms of the parameters of the theory. Indeed, for this ansatz, the $g$ and $f$ equations of motion reduce to two copies Einstein's equation,

$$
R_{\mu \nu}(\bar{g})-\frac{1}{2} \bar{g}_{\mu \nu} R(\bar{g})+\Lambda_{g} \bar{g}_{\mu \nu}=0
$$

and a similar equation, again for $\bar{g}_{\mu \nu}$, but with a cosmological constant $\Lambda_{f}$, where,

$$
\Lambda_{g}=\frac{m^{4}}{m_{g}^{2}}\left(\beta_{0}+3 c \beta_{1}+3 c^{2} \beta_{2}+c^{3} \beta_{3}\right), \quad \Lambda_{f}=\frac{m^{4}}{c^{2} m_{f}^{2}}\left(c \beta_{1}+3 c^{2} \beta_{2}+3 c^{3} \beta_{3}+c^{4} \beta_{4}\right) .
$$

The consistency of the two equations then requires,

$$
\Lambda_{g}=\Lambda_{f} .
$$

This provides, in general, a quartic equation that determines $c$ in terms of the 6 combinations of the seven parameters of the theory,

$$
c=c\left(\alpha, \beta_{n}\right), \quad \text { with }, \quad \alpha \equiv \frac{m_{f}}{m_{g}} .
$$

For the purpose of these solutions, the relevant regions of the parameter space are those that lead to a positive Fierz-Pauli mass for the fluctuation, as given below.

Let us now consider canonically normalized linear perturbations around this background,

$$
g_{\mu \nu}=\bar{g}_{\mu \nu}+\frac{1}{m_{g}} \delta g_{\mu \nu}, \quad f_{\mu \nu}=c^{2} \bar{g}_{\mu \nu}+\frac{1}{m_{f}} \delta f_{\mu \nu} .
$$

The equations of motion for the perturbations are diagonalized in terms of a massless mode $\delta G_{\mu \nu}$ and a massive mode $\delta M_{\mu \nu}$,

$$
\delta G_{\mu \nu}=\frac{\delta g_{\mu \nu}}{m_{g}}+\alpha^{2} \frac{\delta f_{\mu \nu}}{m_{f}}, \quad \delta M_{\mu \nu}=\frac{1+\alpha^{2} c^{2}}{2 c}\left(\frac{\delta f_{\mu \nu}}{m_{f}}-c^{2} \frac{\delta g_{\mu \nu}}{m_{g}}\right) .
$$

The normalizations are explained below. They satisfy the corresponding equations,

$$
\begin{aligned}
& \overline{\mathcal{E}}_{\mu \nu}^{\rho \sigma} \delta G_{\rho \sigma}-\Lambda_{g}\left(\delta G_{\mu \nu}-\frac{1}{2} \bar{g}_{\mu \nu} \bar{g}^{\rho \sigma} \delta G_{\rho \sigma}\right)=0, \\
& \overline{\mathcal{E}}_{\mu \nu}^{\rho \sigma} \delta M_{\rho \sigma}-\Lambda_{g}\left(\delta M_{\mu \nu}-\frac{1}{2} \bar{g}_{\mu \nu} \bar{g}^{\rho \sigma} \delta M_{\rho \sigma}\right)+\frac{1}{2} m_{\mathrm{FP}}^{2}\left(\delta M_{\mu \nu}-\bar{g}_{\mu \nu} \bar{g}^{\rho \sigma} \delta M_{\rho \sigma}\right)=0,
\end{aligned}
$$

\footnotetext{
${ }^{1}$ Simply requiring both $g_{\mu \nu}$ and $f_{\mu \nu}$ to be dS spacetimes restricts the solutions to such proportional backgrounds.
} 
where $\overline{\mathcal{E}}_{\mu \nu}^{\rho \sigma}$ is given by (2.2) and the Fierz-Pauli mass of the massive spin-2 mode reads,

$$
m_{\mathrm{FP}}^{2}=\frac{m^{4}}{m_{g}^{2}}\left(1+\alpha^{-2} c^{-2}\right)\left(c \beta_{1}+2 c^{2} \beta_{2}+c^{3} \beta_{3}\right) .
$$

The normalizations in (3.8) are chosen for convenience so that the mass eigenstates can be regarded as fluctuation of nonlinear modes [33],

$$
G_{\mu \nu}=g_{\mu \nu}+\alpha^{2} f_{\mu \nu}, \quad M_{\mu \nu}=G_{\mu \rho}\left(\sqrt{g^{-1} f}\right)_{\nu}^{\rho}-c G_{\mu \nu} .
$$

Equation (3.10) for the massive spin-2 fluctuation coincides with the Fierz-Pauli equation (2.1). In particular, at the Higuchi bound, $m_{\mathrm{FP}}^{2}=\frac{2}{3} \Lambda_{g}$, it has the same extra gauge invariance (2.4). Now, taking the presence of the massless mode $\delta G_{\mu \nu}$ also into account, the corresponding symmetry transformations in the bimetric theory become,

$$
\delta M_{\mu \nu} \rightarrow \delta M_{\mu \nu}+\left(\nabla_{\mu} \nabla_{\nu}+\frac{\Lambda}{3} \bar{g}_{\mu \nu}\right) \xi(x), \quad \delta G_{\mu \nu} \rightarrow \delta G_{\mu \nu} .
$$

While, superficially, this is very similar to Fierz-Pauli massive gravity, a major difference is that in the bimetric case the background is dynamical and is not fixed by hand. Demanding compatibility between a subset of (3.13) and the dynamical nature of the background is powerful enough to uniquely determine the partially massless nonlinear bimetric theory. This is explained below.

\section{Determination of the partially massless bimetric theory}

From (3.13) and (3.8), one can easily read off the transformations of $\delta g_{\mu \nu}$ and $\delta f_{\mu \nu}$,

$$
\delta g_{\mu \nu} \rightarrow \delta g_{\mu \nu}+a\left(\nabla_{\mu} \nabla_{\nu}+\frac{\Lambda}{3} \bar{g}_{\mu \nu}\right) \xi(x), \quad \delta f_{\mu \nu} \rightarrow \delta f_{\mu \nu}+b\left(\nabla_{\mu} \nabla_{\nu}+\frac{\Lambda}{3} \bar{g}_{\mu \nu}\right) \xi(x),
$$

where the constants $a$ and $b$ are given in terms of $\alpha$ and $c$. The gauge transformations, being symmetries, are trivial solutions of the linearized equations of motion.

The crucial point to note is that, for a dynamical field, say $g_{\mu \nu}$, the split into a background part $\bar{g}_{\mu \nu}$ and a fluctuation $\delta g_{\mu \nu}$ is not unique since, in principle, infinitesimal symmetry transformations can be shifted between the two. Hence, one can always transfer a part of $\delta g_{\mu \nu}$ to the background $\bar{g}_{\mu \nu}$ to get,

$$
g_{\mu \nu}=\bar{g}_{\mu \nu}+\delta g_{\mu \nu}=\bar{g}_{\mu \nu}^{\prime}+\delta g_{\mu \nu}^{\prime},
$$

where the backgrounds $\bar{g}_{\mu \nu}$ and $\bar{g}_{\mu \nu}^{\prime}$ differ by an infinitesimal symmetry transformation.

In bimetric theory, the two metrics are dynamical and come with their own equations of motion. If we assume that the theory has a nonlinear symmetry that manifests itself as (3.13) around the backgrounds considered, then this argument tells us that it should be possible to transfer the infinitesimal transformations (4.1) from the fluctuations to the backgrounds $\bar{g}_{\mu \nu}$ and $\bar{f}_{\mu \nu}$ and end up with new consistent background solutions. But for a generic gauge 
parameter $\xi(x)$ in (4.1), the new $\bar{g}^{\prime}$ and $\bar{f}^{\prime}$ are not proportional and hence are not dS metrics. In this case not much is known about partial masslessness. To have the calculation under control, we keep to dS backgrounds and therefore restrict the transformations in (4.1) to constant $\xi(x)=\xi_{0}$. This subset of (4.1) is consistent with the proportional background ansatz and can be used for the purpose of identifying the PM theory. In this case,

$$
\delta g_{\mu \nu} \rightarrow \delta g_{\mu \nu}+a \frac{\Lambda}{3} \xi \bar{g}_{\mu \nu}, \quad \delta f_{\mu \nu} \rightarrow \delta f_{\mu \nu}+b \frac{\Lambda}{3} \xi \bar{g}_{\mu \nu} .
$$

Transferring these from the fluctuations to the backgrounds gives,

$$
\bar{g}_{\mu \nu}^{\prime}=\bar{g}_{\mu \nu}+a \frac{\Lambda}{3} \xi \bar{g}_{\mu \nu}, \quad \bar{f}_{\mu \nu}^{\prime}=\bar{f}_{\mu \nu}+b \frac{\Lambda}{3} \xi \bar{g}_{\mu \nu}
$$

Now, it is obvious that $f^{\prime}=c^{\prime 2} g^{\prime}$ with a constant $c^{\prime}(\xi)$, and $c^{\prime} \neq c$. As such $c^{\prime}$ will differ from $c$ infinitesimally. However, if we insure that $\bar{g}_{\mu \nu}^{\prime}$ and $\bar{f}_{\mu \nu}^{\prime}$ are exact dS solutions (not just to linear order in $\delta c=c^{\prime}-c$ ), then perturbations around the new backgrounds are again invariant under (4.1) and the process can be repeated to generate a finite transformation. In other words, we require that transformations (4.4) are integrable in the nonlinear theory and can be iterated to generate finite $\delta c$ (the integrated form of (4.4) is given in the next section).

On the other hand, from the previous section we know that, generically, the bimetric equations fix $c$ in terms of the parameters of the theory and hence $c^{\prime}(\xi)$ cannot be a solution to (3.5). The obvious implication is that, for generic bimetric parameters, $\bar{g}_{\mu \nu}^{\prime}$ and $\bar{f}_{\mu \nu}^{\prime}$ are not valid background solutions. This can happen only if the transformations that generate them are not symmetries of the nonlinear theory. Thus the only parameter values for which the bimetric theory is consistent with the transformations (4.3), are those for which the equation for $c$ (3.5) does not determine $c$ at all! This is the necessary condition for the existence of a nonlinear partially massless bimetric theory.

Note that by fixing $\xi(x)$ to be constant, we restrict ourselves to only part of the gauge group. If in fact the full gauge symmetry is realized at the nonlinear level, the theory obtained from using only the subgroup will definitely contain the partially massless theory invariant under the full symmetry.

Having established a necessary condition for the existence of the nonlinear partially massless theory, one can easily find the parameter values that leave the $c$ in the proportional background ansatz undetermined. The equation (3.5) that determines $c$ can be written as,

$$
\beta_{1}+\left(3 \beta_{2}-\alpha^{2} \beta_{0}\right) c+\left(3 \beta_{3}-3 \alpha^{2} \beta_{1}\right) c^{2}+\left(\beta_{4}-3 \alpha^{2} \beta_{2}\right) c^{3}-\alpha^{2} \beta_{3} c^{4}=0 .
$$

Thus the parameter combination for which $c$ remains undetermined is,

$$
\alpha^{2} \beta_{0}=3 \beta_{2}, \quad 3 \alpha^{2} \beta_{2}=\beta_{4}, \quad \beta_{1}=\beta_{3}=0 .
$$

Remarkably, this fixes all but one of the $\beta_{n}$ which immediately implies that if there is a nonlinear partially massless theory it has to be this one. Note that with the choice (4.6) we have

$$
m_{\mathrm{FP}}^{2}=2 \frac{m^{4}}{m_{g}^{2}}\left(\alpha^{-2}+c^{2}\right) \beta_{2}=\frac{2}{3} \Lambda_{g},
$$


so in particular the massive fluctuation has a mass at the Higuchi bound.

As a side remark we note that the PM action is symmetric under $\alpha^{-1} g_{\mu \nu} \leftrightarrow \alpha f_{\mu \nu}$ which is a consequence of $\sqrt{g} e_{n}(S)=\sqrt{f} e_{4-n}\left(S^{-1}\right)$ [21]. While this interchange is a symmetry of (3.1) whenever the parameters satisfy $\alpha^{-n} \beta_{n}=\alpha^{n-4} \beta_{4-n}$, the PM case corresponds to a further restriction to a single parameter.

The reasoning presented here is simple and straightforward enough that it can be easily generalized to any number of dimensions and to theories with multiple spin-2 fields [34, 35].

\section{$5 \quad$ Nonlinear scaling symmetry}

Taking into account the value for $\Lambda_{g}$ given in (4.7), it is easy to see that for the parameter choice (4.6) the background equations (3.3) are invariant under the simultaneous transformations,

$$
c \longrightarrow c+a, \quad \bar{g}_{\mu \nu} \longrightarrow \frac{\alpha^{-2}+c^{2}}{\alpha^{-2}+(c+a)^{2}} \bar{g}_{\mu \nu}, \quad a \in \mathbb{R} .
$$

This is the nonlinear version of (4.4) and verifies that the transformations considered are indeed integrable as required. The linearized versions of (5.1) read

$$
c \longrightarrow c+\delta c, \quad \bar{g}_{\mu \nu} \longrightarrow \bar{g}_{\mu \nu}+\delta \bar{g}_{\mu \nu} \equiv \bar{g}_{\mu \nu}-\frac{2 c}{\alpha^{-2}+c^{2}} \delta c \bar{g}_{\mu \nu} .
$$

Moreover, using $\bar{f}_{\mu \nu}=c^{2} \bar{g}_{\mu \nu}$, we find that $\bar{f}_{\mu \nu}$ transforms as

$$
\bar{f}_{\mu \nu} \longrightarrow \bar{f}_{\mu \nu}+\delta \bar{f}_{\mu \nu} \equiv \bar{f}_{\mu \nu}+2 c \delta c \bar{g}_{\mu \nu}+c^{2} \delta \bar{g}_{\mu \nu} .
$$

We will now reverse the arguments that were given in section 4 and translate these scalings of the backgrounds into transformations of the fluctuations in order to see if we can re-arrive at the $\xi=$ const. version of the transformation (3.13) for the massive fluctuation in de Sitter space. For this we identify $\delta f_{\mu \nu}=m_{f} \delta \bar{f}_{\mu \nu}$ and $\delta g_{\mu \nu}=m_{g} \delta \bar{g}_{\mu \nu}$. Then we see that the massless and massive fluctuation transform as

$$
\delta G_{\mu \nu} \longrightarrow \delta G_{\mu \nu}, \quad \delta M_{\mu \nu} \longrightarrow \delta M_{\mu \nu}+\frac{\Lambda_{g}}{3} \frac{m_{f}^{2}}{\beta_{2} m^{4}} \bar{g}_{\mu \nu} \delta c
$$

where we have used (4.7) to express the transformation of $\delta M_{\mu \nu}$ in terms of $\Lambda_{g}$. As required, the symmetry transformation (5.2) leaves the massless fluctuation invariant ${ }^{2}$ while the massive fluctuation transforms as under a scaling. The transformation of $\delta M_{\mu \nu}$ is precisely of the form (3.13) with $\xi=\frac{m_{f}^{2}}{\beta_{2} m^{4}} \delta c$. Thus we have reproduced the linearized symmetry with constant gauge parameter known to be present at the Higuchi bound through the constant but nonlinear transformation (5.1).

We emphasize once more that all of these conclusions become invalid once we choose parameters different from (4.6) since then the background equations do determine $c$ and there is no invariance at the background level. Thus the unique candidate for a nonlinear partially massless theory is the one specified by (4.6).

\footnotetext{
${ }^{2}$ Note that, in addition, the background $\bar{G}_{\mu \nu}=\left(1+\alpha^{2} c^{2}\right) \bar{g}_{\mu \nu}$ of the nonlinear massless field $G_{\mu \nu}=$ $g_{\mu \nu}+\alpha^{2} f_{\mu \nu}$ is invariant under the full nonlinear scaling symmetry (5.1).
} 


\section{$6 \quad$ Summary and Discussions}

Ghost-free bimetric theories admit de Sitter backgrounds with Fierz-Pauli massive spin-2 excitations. A large subclass of these theories can easily satisfy the Higuchi bound and exhibit partial masslessness at the linear level. However, using simple symmetry arguments, we have shown that it is possible to identify a unique theory in this multi-parameter space as the only possible candidate for a non-linear PM theory. The construction involves showing that (i) the known linear PM transformations contain a simple de Sitter preserving subgroup generated by constant gauge parameter $\xi_{0}$, and that (ii) in any non-linear PM setup it must be possible to integrate such linear dS-preserving transformations to a non-linear one. This is the necessary condition that must be satisfied by any non-linear PM theory, if such a theory exists. In the bimetric setup in 4-dimensions, this singles out a unique theory specified by (4.6) as the only possible candidate for a non-linear PM theory. While the construction narrows down the search to a unique candidate, it does not prove that it actually has a PM symmetry. This may or may not be the case and the proof is beyond the scope of this paper.

While this paper focuses on bimetric theories, our logic and construction is general and can be applied to any nonlinear setup that accommodates linear PM symmetry around dS backgrounds to identify the potential non-linear PM candidates.

In [23], the authors identify a specific nonlinear massive gravity which, in a decoupling limit adapted to dS spacetimes, shows a non-linear PM symmetry. To compare results, we need to take a massive gravity limit of (4.6) by setting $m_{f}=\infty$ (or $\alpha=\infty$ ) keeping $\beta_{2}$ fixed. This gives $\beta_{0}=0$ and $\beta_{4}=\alpha^{2} \beta_{4}^{\prime}$ for a finite $\beta_{4}^{\prime}$, agreeing with the parameters for which [23] finds an extra PM symmetry in the decoupling limit ${ }^{3}$, although, in general the two formalisms lead to different predictions, as described below.

An important question that remains unanswered here is, if the candidate non-linear theory can really have the full PM gauge symmetry, beyond the dS preserving subset used here. Recent evidence for and against this will be discussed below. Here we point out that the theory specified by (4.6) definitely has an extra nonlinear gauge symmetry for one class of non-proportional backgrounds. Consider non-proportional homogeneous and isotropic backgrounds parameterized by three functions $a(t), Y(t)$ and $X(t)$ [36],

$$
g_{\mu \nu} \mathrm{d} x^{\mu} \mathrm{d} x^{\nu}=-\mathrm{d} t^{2}+a^{2}(t) \mathrm{d} \vec{x}^{2}, \quad f_{\mu \nu} \mathrm{d} x^{\mu} \mathrm{d} x^{\nu}=-X^{2}(t) \mathrm{d} t^{2}+Y^{2}(t) \mathrm{d} \vec{x}^{2}
$$

The bimetric equations can in general be solved to determine the three functions. The fluctuations around these backgrounds do not have the Fierz-Pauli form. However, for the parameter values specified by (4.6), and in the absence of sources, the equation that determines $\Upsilon=Y / a$

\footnotetext{
${ }^{3}$ Comparison with the theory found in [23] requires some care. The massive gravity action presented there contains the original dRGT potential which is constructed with $\alpha_{1}=0$ so that the $g_{\mu \nu}=f_{\mu \nu}$ solutions have zero cosmological constant. Using this action one reads off $\beta_{1}=\beta_{3}=0, \beta_{2}=-\frac{1}{2}$ and $\beta_{0}=\frac{3}{2} \neq 0$ (for example, using the relations in appendix B of [36]). However, to find the PM symmetry, [23] considers perturbations around $g_{\mu \nu}=f_{\mu \nu}$ backgrounds with a de Sitter $f_{\mu \nu}$. The correct action corresponding to this must have $\alpha_{1} \neq 0$ such that $\beta_{0}=0$, consistent with the massive gravity limit of the theory found here.
} 
disappears, as can be verified from [36]. Hence the theory leaves one of the three functions in (6.1) undetermined. This implies a nonlinear gauge symmetry of the cosmological metrics (6.1) in the candidate PM theory, well beyond the linear PM symmetry (4.1) around proportional backgrounds, albeit only for a cosmological gauge parameter. It is also interesting to understand the partially massless bimetric theory in connection with the self-protection mechanism discussed in [37-40].

Since this work first appeared, several papers have addressed the issue of PM symmetry in nonlinear massive gravity and in bimetric theory, providing arguments both in favour and against it. Below we briefly comment on these developments. [43] provides evidence for the strength of the method used here by applying it to general dimensions. It finds that PM theories cannot exist for $d>4$ in a 2-derivative theory, consistent with perturbative reasoning. This differs from the prediction in [23] which is not dimension sensitive. Furthermore, [44] finds evidence for an extra gauge symmetry in the candidate PM theory by partially solving the bimetric equations perturbatively in powers of curvatures of $g_{\mu \nu}$. Then, at the 4-derivative level, the PM bimetric equations coincide with the conformal gravity equation of motion which propagates 6 , rather than 7 , modes. Then, to this order, the PM symmetry is related to the Weyl scaling of $g_{\mu \nu}$. The relation to conformal gravity already shows the special status of the theory identified by (4.6), irrespective of the final fate of the PM symmetry.

On the other hand, [41, 42] argue against the existence of PM symmetry in massive gravity. Specifically, [41] cannot find a PM symmetry in the massive gravity action (which it takes as (3.1) without the $\sqrt{f} R(f)$ term) while [42] cannot find the associated Binachi identities. While these studies are limited to massive gravity, one may wonder if the claimed absence of a PM massive gravity could already rule out a PM bimetric theory. If the PM bimetric theory happens to have a well behaved massive gravity limit, then one expects its PM symmetry to survive in the limit. If so, the absence of a PM massive gravity would also imply the absence of a PM bimetric theory. While this is not ruled out at this stage, there are indications that the massive gravity limit of the PM bimetric theory is not completely well behaved in the sense that not all bimetric solutions have a massive gravity limit.

Massive gravity is obtained by setting $m_{f}=\infty$ in the bimetric equations, then the $g_{\mu \nu}$ equation is unchanged, while the $f_{\mu \nu}$ equation reduces to an Einstein equation,

$$
m_{g}^{2} G_{\mu \nu}(g)+V_{\mu \nu}^{g}=0, \quad G_{\mu \nu}(f)+\beta_{4}^{\prime} f_{\mu \nu}=0,
$$

where $G_{\mu \nu}$ denotes the Einstein tensor and $\beta_{4}^{\prime}=\beta_{4} / m_{f}^{2}$ is fixed. These two equations can no longer be obtained from an action principle. If the PM gauge transformation of bimetric theory depends non-trivially on $m_{f}$, it will not survive on setting $m_{f}=\infty$. The bimetric solutions generated by such transformations are the ones that do not have a massive gravity limit. Using the perturbative solution for $f_{\mu \nu}$ in powers of curvatures of $g_{\mu \nu}$, found in [44], one can check that the transformation of $f_{\mu \nu}$ induced by a Weyl scaling of $g_{\mu \nu}$ contains terms that diverge for $m_{f}=\infty$, but which vanish for a dS (or Einstein) $g_{\mu \nu}$. This leads one to expect that the gauge symmetry of the candidate PM bimetric theory is lost in the massive gravity, except around dS backgrounds. 
Another difference can be seen at the level of classical solutions. In massive gravity, where $f_{\mu \nu}$ is always an Einstein metric, the fluctuation spectrum around $g_{\mu \nu} \propto f_{\mu \nu}$ can have a PM symmetry. But, it is also possible to find a $g_{\mu \nu}$ background that is not an Einstein metric and around these PM symmetry is evidently lost. On the contrary, in the candidate bimetric PM theory, one can show that whenever $f_{\mu \nu}$ is an Einstein metric then $g_{\mu \nu}$ is necessarily a proportional Einstein metric in which case the linear spectrum always has a PM symmetry. Thus, for any finite $m_{f}$, it is impossible to find PM violating massive gravity type backgrounds where only $f_{\mu \nu}$ (but not $g_{\mu \nu}$ ), is an Einstein metric. This constraint disappears precisely for $m_{f}=\infty$, in which case PM violating backgrounds become allowed classical solutions. Finally, it is likely that a PM symmetry exists only on-shell and not at the level of the action. The above arguments indicate that the massive gravity investigations in [41, 42] do not necessarily rule out a PM bimetric theory.

Acknowledgments: We would like to thank Augusto Sagnotti for asking the questions that led us to the present investigation.

\section{References}

[1] W. Pauli and M. Fierz, Helv. Phys. Acta 12 (1939) 297.

[2] M. Fierz and W. Pauli, Proc. Roy. Soc. Lond. A 173 (1939) 211.

[3] A. Higuchi, Nucl. Phys. B 282 (1987) 397.

[4] A. Higuchi, Nucl. Phys. B 325 (1989) 745.

[5] S. Deser and R. I. Nepomechie, Annals Phys. 154 (1984) 396.

[6] S. Deser and A. Waldron, Phys. Rev. Lett. 87 (2001) 031601 [hep-th/0102166].

[7] S. Deser and A. Waldron, Nucl. Phys. B 607 (2001) 577 [hep-th/0103198].

[8] S. Deser and A. Waldron, Phys. Lett. B 508 (2001) 347 [hep-th/0103255].

[9] S. Deser and A. Waldron, Phys. Lett. B 513 (2001) 137 [hep-th/0105181].

[10] S. Deser and A. Waldron, Phys. Lett. B 603 (2004) 30 [hep-th/0408155].

[11] S. Deser and A. Waldron, Phys. Rev. D 74 (2006) 084036 [hep-th/0609113].

[12] D. G. Boulware and S. Deser, Phys. Lett. B 40 (1972) 227.

[13] D. G. Boulware and S. Deser, Phys. Rev. D 6 (1972) 3368.

[14] C. de Rham and G. Gabadadze, Phys. Rev. D 82 (2010) 044020 [arXiv:1007.0443 [hep-th]].

[15] C. de Rham, G. Gabadadze and A. J. Tolley, Phys. Rev. Lett. 106 (2011) 231101 [arXiv:1011.1232 [hep-th]].

[16] N. Arkani-Hamed, H. Georgi and M. D. Schwartz, Annals Phys. 305 (2003) 96 [hep-th/0210184].

[17] P. Creminelli, A. Nicolis, M. Papucci and E. Trincherini, JHEP 0509 (2005) 003 [hep-th/0505147]. 
[18] S. F. Hassan and R. A. Rosen, Phys. Rev. Lett. 108 (2012) 041101 [arXiv:1106.3344 [hep-th]].

[19] S. F. Hassan, R. A. Rosen, JHEP 1107 (2011) 009. [arXiv:1103.6055 [hep-th]].

[20] S. F. Hassan, R. A. Rosen and A. Schmidt-May, JHEP 1202 (2012) 026 [arXiv:1109.3230 [hep-th]].

[21] S. F. Hassan and R. A. Rosen, JHEP 1202 (2012) 126 [arXiv:1109.3515 [hep-th]].

[22] S. F. Hassan and R. A. Rosen, JHEP 1204 (2012) 123 [arXiv:1111.2070 [hep-th]].

[23] C. de Rham and S. Renaux-Petel, arXiv:1206.3482 [hep-th].

[24] M. Fasiello and A. J. Tolley, arXiv:1206.3852 [hep-th].

[25] A. Iglesias and Z. Kakushadze, Phys. Rev. D 82 (2010) 124001 [arXiv:1007.2385 [hep-th]].

[26] S. Deser, E. Joung and A. Waldron, arXiv:1208.1307 [hep-th].

[27] O. Hohm, A. Routh, P. K. Townsend and B. Zhang, arXiv:1208.0038 [hep-th].

[28] E. A. Bergshoeff, O. Hohm and P. K. Townsend, Phys. Rev. D 79 (2009) 124042 [arXiv:0905.1259 [hep-th]].

[29] E. A. Bergshoeff, O. Hohm and P. K. Townsend, Phys. Rev. Lett. 102 (2009) 201301 [arXiv:0901.1766 [hep-th]].

[30] A. Sagnotti, arXiv:1112.4285 [hep-th].

[31] D. Francia, J. Mourad and A. Sagnotti, Nucl. Phys. B 804 (2008) 383 [arXiv:0803.3832 [hep-th]].

[32] E. Joung, L. Lopez and M. Taronna, JHEP 1207 (2012) 041 [arXiv:1203.6578 [hep-th]].

[33] S. F. Hassan, A. Schmidt-May, and M. von Strauss, [arXiv:1208.1515 [hep-th]].

[34] K. Hinterbichler and R. A. Rosen, JHEP 1207 (2012) 047 [arXiv:1203.5783 [hep-th]].

[35] S. F. Hassan, A. Schmidt-May and M. von Strauss, arXiv:1204.5202 [hep-th].

[36] M. von Strauss, A. Schmidt-May, J. Enander, E. Mortsell and S. F. Hassan, JCAP 1203 (2012) 042 [arXiv:1111.1655 [gr-qc]].

[37] L. Grisa and L. Sorbo, Phys. Lett. B 686 (2010) 273 [arXiv:0905.3391 [hep-th]].

[38] F. Berkhahn, D. D. Dietrich and S. Hofmann, JCAP 1011 (2010) 018 [arXiv:1008.0644 [hep-th]].

[39] F. Berkhahn, D. D. Dietrich and S. Hofmann, Phys. Rev. Lett. 106 (2011) 191102 [arXiv:1102.0313 [hep-th]].

[40] F. Berkhahn, D. D. Dietrich and S. Hofmann, JCAP 1109 (2011) 024 [arXiv:1104.2534 [hep-th]].

[41] C. de Rham, K. Hinterbichler, R. A. Rosen and A. J. Tolley, arXiv:1302.0025 [hep-th].

[42] S. Deser, M. Sandora and A. Waldron, arXiv:1301.5621 [hep-th].

[43] S. F. Hassan, A. Schmidt-May and M. von Strauss, arXiv:1212.4525 [hep-th].

[44] S. F. Hassan, A. Schmidt-May and M. von Strauss, arXiv:1303.6940 [hep-th]. 